\title{
Independent Component Analysis Using an Extended Infomax Algorithm for Mixed Subgaussian and Supergaussian Sources
}

\section{Te-Won Lee}

Howard Hughes Medical Institute, Computational Neurobiology Laboratory, Salk Institute, La Jolla, CA 92037, U.S.A., and

Institut für Electronik, Technische Universität Berlin, Berlin, Germany

\section{Mark Girolami}

Department of Computing and Information Systems, University of Paisley, PA1 2BE, Scotland

\section{Terrence J. Sejnowski}

Howard Hughes Medical Institute, Computational Neurobiology Laboratory, Salk Institute, La Jolla, CA 92037, U.S.A., and

Department of Biology, University of California, San Diego, La Jolla, CA 92093, U.S.A.

An extension of the infomax algorithm of Bell and Sejnowski (1995) is presented that is able blindly to separate mixed signals with sub- and supergaussian source distributions. This was achieved by using a simple type of learning rule first derived by Girolami (1997) by choosing negentropy as a projection pursuit index. Parameterized probability distributions that have sub- and supergaussian regimes were used to derive a general learning rule that preserves the simple architecture proposed by Bell and Sejnowski (1995), is optimized using the natural gradient by Amari (1998), and uses the stability analysis of Cardoso and Laheld (1996) to switch between sub- and supergaussian regimes. We demonstrate that the extended infomax algorithm is able to separate 20 sources with a variety of source distributions easily. Applied to high-dimensional data from electroencephalographic recordings, it is effective at separating artifacts such as eye blinks and line noise from weaker electrical signals that arise from sources in the brain.

\section{Introduction}

Recently, blind source separation by independent component analysis (ICA) has received attention because of its potential signal processing applications, such as speech enhancement systems, telecommunications, and medical signal processing. The goal of ICA is to recover independent sources given only sensor observations that are unknown linear mixtures of the unobserved independent source signals. In contrast to correlation-based transformations 
such as principal component analysis (PCA), ICA reduces the statistical dependencies of the signals, attempting to make the signals as independent as possible.

The blind source separation problem has been studied by many researchers in neural networks and statistical signal processing (Jutten \& Hérault, 1991; Comon, 1994; Cichocki, Unbehauen, \& Rummert, 1994; Bell \& Sejnowski, 1995; Cardoso \& Laheld, 1996; Amari, Cichocki, \& Yang, 1996; Pearlmutter \& Parra, 1996; Deco \& Obradovic, 1996; Oja, 1997; Karhunen, Oja, Wang, Vigario, \& Joutsensalo, 1997; Girolami \& Fyfe, 1997a). See the introduction of Nadal and Parga (1997) for a historical review of ICA, and Karhunen (1996) for a review of different neural-based blind source separation algorithms. More general ICA reviews are in Cardoso (1998), Lee (1998), \& Lee, Girolami, Bell, \& Sejnowski (1999).

Bell and Sejnowski (1995) have developed an unsupervised learning algorithm based on entropy maximization in a single-layer feedforward neural network. The algorithm is effective in separating sources that have supergaussian distributions: sharply peaked probability density functions (p.d.f.s) with heavy tails. As illustrated in section 4 of Bell and Sejnowski (1995), the algorithm fails to separate sources that have negative kurtosis (e.g., uniform distribution). Pearlmutter and Parra (1996) have developed a contextual ICA algorithm within the maximum likelihood estimation (MLE) framework that is able to separate a more general range of source distributions. Motivated by computational simplicity, we use an information-theoretic algorithm that preserves the simple architecture in Bell and Sejnowski (1995) and allows an extension to the separation of mixtures of supergaussian and subgaussian sources. Girolami (1997) derived this type of learning rule from the viewpoint of negentropy maximization ${ }^{1}$ for exploratory projection pursuit (EPP) and ICA. These algorithms can be used on-line as well as off-line. Off-line algorithms that can also separate mixtures of supergaussian and subgaussian sources were proposed by Cardoso and Soloumiac (1993), Comon (1994), and Pham and Garrat (1997).

The extended infomax algorithm preserves the simple architecture in Bell and Sejnowski (1995) and the learning rule converges rapidly with the "natural" gradient proposed by Amari et al. (1996) and Amari (1998) or the "relative" gradient proposed by Cardoso and Laheld (1996). In computer simulations, we show that this algorithm can successfully separate 20 mixtures of the following sources: 10 soundtracks ${ }^{2}, 6$ speech and sound signals used in Bell and Sejnowski (1995), 3 uniformly distributed subgaussian noise signals, and 1 noise source with a gaussian distribution. To test the extended infomax algorithm on more challenging real-world data, we

\footnotetext{
${ }^{1}$ Relative entropy is the general term for negentropy. Negentropy maximization refers to maximizing the sum of marginal negentropies.

${ }^{2}$ Obtained from Pearlmutter online at http://sweat.cs.unm.edu/ bap/demos.html.
} 
performed experiments with electroencephalogram (EEG) recordings and show that it can clearly separate electrical artifacts from brain activity. This technique shows great promise for analyzing EEG recordings (Makeig, Jung, Bell, Ghahremani, \& Sejnowski, 1997; Jung et al., 1998) and functional magnetic resonance imaging (fMRI) data (McKeown et al., 1998).

In section 2 , the problem is stated and a simple but general learning rule that can separate sub- and supergaussian sources is presented. This rule is applied to simulations and real data in section 3. Section 4 contains a brief discussion of other algorithms and architectures, potential applications to real-world problems, limitations, and further research problems.

\section{The Extended Infomax Algorithm}

Assume that there is an $M$-dimensional zero-mean vector $\mathbf{s}(t)=\left[s_{1}(t), \ldots\right.$, $\left.s_{M}(t)\right]^{T}$, such that the components $s_{i}(t)$ are mutually independent. The vector $\mathbf{s}(t)$ corresponds to $M$ independent scalar-valued source signals $s_{i}(t)$. We can write the multivariate p.d.f. of the vector as the product of marginal independent distributions:

$$
p(\mathbf{s})=\prod_{i=1}^{M} p_{i}\left(s_{i}\right) .
$$

A data vector $\mathbf{x}(t)=\left[x_{1}(t), \ldots, x_{N}(t)\right]^{T}$ is observed at each time point $t$, such that

$$
\mathbf{x}(t)=\mathbf{A s}(t)
$$

where A is a full-rank $N \times M$ scalar matrix. Because the components of the observed vectors are no longer independent, the multivariate p.d.f. will not satisfy the p.d.f. product equality. In this article, we shall consider the case where the number of sources is equal to the number of sensors $N=M$. If the components of $\mathbf{s}(t)$ are such that at most one source is normally distributed, then it is possible to extract the sources $\mathbf{s}(t)$ from the received mixtures $\mathbf{x}(t)$ (Comon, 1994). The mutual information of the observed vector is given by the Kullback-Leibler (KL) divergence of the multivariate density from the product of the marginal (univariate) densities:

$$
\begin{aligned}
I\left(x_{1}, x_{2}, \ldots, x_{N}\right)= & \int_{-\infty}^{+\infty} \int_{-\infty}^{+\infty} \cdots \int_{-\infty}^{+\infty} p\left(x_{1}, x_{2}, \ldots, x_{N}\right) \\
& \times \log \frac{p\left(x_{1}, x_{2}, \ldots, x_{N}\right)}{\prod_{i=1}^{N} p_{i}\left(x_{i}\right)} d x_{1} d x_{2}, \ldots, d x_{N} .
\end{aligned}
$$


For simplicity, we write:

$$
I(\mathbf{x})=\int p(\mathbf{x}) \log \frac{p(\mathbf{x})}{\prod_{i=1}^{N} p_{i}\left(x_{i}\right)} d \mathbf{x} .
$$

The mutual information will always be positive and will equal zero only when the components are independent (Cover \& Thomas, 1991).

The goal of ICA is to find a linear mapping $\mathbf{W}$ such that the unmixed signals $\mathbf{u}$,

$$
\mathbf{u}(t)=\mathbf{W} \mathbf{x}(t)=\mathbf{W A s}(t),
$$

are statistically independent. The sources are recovered up to scaling and permutation. There are many ways for learning W. Comon (1994) minimizes the degree of dependence among outputs using contrast functions approximated by the Edgeworth expansion of the KL divergence. The higher-order statistics are approximated by cumulants up to fourth order. Other methods related to minimizing mutual information can be derived from the infomax approach. Nadal and Parga (1994) showed that in the low-noise case, the maximum of the mutual information between the input and output of a neural processor implied that the output distribution was factorial. Roth and Baram (1996) and Bell and Sejnowski (1995) independently derived stochastic gradient learning rules for this maximization and applied them, respectively, to forecasting, time-series analysis, and the blind separation of sources. A similar adaptive method for source separation has been proposed by Cardoso and Laheld (1996).

2.1 A Simple But General Learning Rule. The learning algorithm can be derived using the maximum likelihood formulation. The MLE approach to blind source separation was first proposed by Gaeta and Lacoume (1990) and Pham and Garrat (1997) and was pursued more recently by Pearlmutter and Parra (1996) and Cardoso (1997). The p.d.f. of the observations $\mathbf{x}$ can be expressed as (Amari \& Cardoso, 1997):

$$
p(\mathbf{x})=|\operatorname{det}(\mathbf{W})| p(\mathbf{u})
$$

where $p(\mathbf{u})=\prod_{i=1}^{N} p_{i}\left(u_{i}\right)$ is the hypothesized distribution of $p(\mathbf{s})$. The loglikelihood of equation 2.6 is

$$
L(\mathbf{u}, \mathbf{W})=\log |\operatorname{det}(\mathbf{W})|+\sum_{i=1}^{N} \log p_{i}\left(u_{i}\right) .
$$

Maximizing the log-likelihood with respect to $\mathbf{W}$ gives a learning algorithm for W (Bell \& Sejnowski, 1995):

$$
\Delta \mathbf{W} \propto\left[\left(\mathbf{W}^{T}\right)^{-1}-\varphi(\mathbf{u}) \mathbf{x}^{T}\right],
$$


where

$$
\varphi(\mathbf{u})=-\frac{\frac{\partial p(\mathbf{u})}{\partial \mathbf{u}}}{p(\mathbf{u})}=\left[-\frac{\frac{\partial p\left(u_{1}\right)}{\partial u_{1}}}{p\left(u_{1}\right)}, \ldots,-\frac{\frac{\partial p\left(u_{N}\right)}{\partial u_{N}}}{p\left(u_{N}\right)}\right]^{T} .
$$

An efficient way to maximize the log-likelihood is to follow the "natural" gradient (Amari, 1998),

$$
\Delta \mathbf{W} \propto \frac{\partial L(\mathbf{u}, \mathbf{W})}{\partial \mathbf{W}} \mathbf{W}^{T} \mathbf{W}=\left[\mathbf{I}-\varphi(\mathbf{u}) \mathbf{u}^{T}\right] \mathbf{W}
$$

as proposed by Amari et al. (1996), or the relative gradient, proposed by Cardoso and Laheld (1996). Here $\mathbf{W}^{T} \mathbf{W}$ rescales the gradient, simplifies the learning rule in equation 2.8 , and speeds convergence considerably. It has been shown that the general learning algorithm in equation 2.10 can be derived from several theoretical viewpoints such as MLE (Pearlmutter \& Parra, 1996), infomax (Bell \& Sejnowski, 1995), and negentropy maximization (Girolami \& Fyfe, 1997b). Lee, Girolami, Bell, \& Sejnowski, in press, review these techniques and show their relation to each other.

The parametric density estimate $p_{i}\left(u_{i}\right)$ plays an essential role in the success of the learning rule in equation 2.10. Local convergence is ensured if $p_{i}\left(u_{i}\right)$ is the derivative of the log densities of the sources (Pham \& Garrat, 1997). If we choose $g_{i}(u)$ to be a logistic function $\left(g_{i}\left(u_{i}\right)=\tanh \left(u_{i}\right)\right)$ so that $\varphi(\mathbf{u})=2 \tanh (\mathbf{u})$, the learning rule reduces to that in Bell and Sejnowski (1995) with the natural gradient:

$$
\Delta \mathbf{W} \propto\left[\mathbf{I}-2 \tanh (\mathbf{u}) \mathbf{u}^{T}\right] \mathbf{W}
$$

Theoretical considerations as well as empirical observations ${ }^{3}$ have shown that this algorithm is limited to separating sources with supergaussian distributions. The sigmoid function used in Bell and Sejnowski (1995) provides a priori knowledge about the source distribution, that is, the supergaussian shape of the sources. However, they also discuss a "flexible" sigmoid function (a sigmoid function with parameters $p, r$ so that $g\left(u_{i}\right)=$ $\left.\int g\left(u_{i}\right)^{p}\left(1-g\left(u_{i}\right)\right)^{r}\right)$ can be used to match the source distribution. The idea of modeling a parametric nonlinearity has been further investigated and generalized by Pearlmutter and Parra (1996) in their contextual ICA (cICA) algorithm. They model the p.d.f. in a parametric form by taking into account the temporal information and choosing $p_{i}\left(u_{i}\right)$ as a weighted sum of several logistic density functions with variable means and scales. Moulines, Cardoso, and Cassiat (1997) and Xu, Cheung, Yang, and Amari (1997) model the underlying p.d.f. with mixtures of gaussians and show that they can

\footnotetext{
${ }^{3}$ As detailed in section 4 of Bell and Sejnowski (1995).
} 
separate sub- and supergaussian sources. These parametric modeling approaches are in general computationally expensive. In addition, our empirical results on EEG and event-related potentials (ERP) using contextual ICA indicate that cICA can fail to find independent components. Our conjecture is that this is due to the limited number of recorded time points (e.g., 600 data points for ERPs) from which a reliable density estimate is difficult.

2.2 Deriving a Learning Rule to Separate Sub- and Supergaussian Sources. The purpose of the extended infomax algorithm is to provide a simple learning rule with a fixed nonlinearity that can separate sources with a variety of distributions. One way of generalizing the learning rule to sources with either sub- or supergaussian distributions is to approximate the estimated p.d.f. with an Edgeworth expansion or Gram-Charlier expansion (Stuart \& Ord, 1987), as proposed by Girolami and Fyfe (1997b). Girolami (1997) used a parametric density estimate to derive the same learning rule without making any approximations, as we show below.

A symmetric strictly subgaussian density can be modeled using a symmetrical form of the Pearson mixture model (Pearson, 1894) as follows (Girolami, 1997, 1998):

$$
p(u)=\frac{1}{2}\left(N\left(\mu, \sigma^{2}\right)+N\left(-\mu, \sigma^{2}\right)\right),
$$

where $N\left(\mu, \sigma^{2}\right)$ is the normal density with mean $\mu$ and variance $\sigma^{2}$. Figure 1 shows the form of the density $p(u)$ for $\sigma^{2}=1$ with varying $\mu=[0 \cdots 2]$. For $\mu=0 p(u)$ is a gaussian model whereas for $\mu_{i}=1.5$, for example, the $p(u)$ is clearly bimodal. The kurtosis $k_{4}$ (normalized fourth-order cumulant) of $p(u)$ is

$$
\kappa=\frac{c_{4}}{c_{2}^{2}}=\frac{-2 \mu^{4}}{\left(\mu^{2}+\sigma^{2}\right)^{2}}
$$

where $c_{i}$ is the $i$ th-order cumulant (Girolami,1997). Depending on the values of $\mu$ and $\sigma^{2}$, the kurtosis lies between -2 and 0 . So equation 2.12 defines a strictly subgaussian symmetric density when $\mu>0$. Defining $a=\frac{\mu}{\sigma^{2}}$ and applying equation 2.12 , we may write for $\varphi(u)$

$$
\varphi(u)=-\frac{\frac{\partial p(u)}{\partial u}}{p(u)}=\frac{u}{\sigma^{2}}-a\left(\frac{\exp (a u)-\exp (-a u)}{\exp (a u)+\exp (-a u)}\right) .
$$

Using the definition of the hyperbolic tangent, we can write

$$
\varphi(u)=\frac{u}{\sigma^{2}}-\frac{\mu}{\sigma^{2}} \tanh \left(\frac{\mu}{\sigma^{2}} u\right) .
$$


Setting $\mu=1$ and $\sigma^{2}=1$, equation 2.15 reduces to

$$
\varphi(u)=u-\tanh (u) .
$$

The learning rule for strictly subgaussian sources is now (equations 2.10 and 2.16)

$$
\Delta \mathbf{W} \propto\left[\mathbf{I}+\tanh (\mathbf{u}) \mathbf{u}^{T}-\mathbf{u} \mathbf{u}^{T}\right] \mathbf{W} .
$$

In the case of unimodal supergaussian sources, we adopt the following density model

$$
p(u) \propto p_{G}(u) \operatorname{sech}^{2}(u),
$$

where $p_{G}(u)=N(0,1)$ is a zero-mean gaussian density with unit variance. Figure 2 shows the density model for $p(u)$. The nonlinearity $\varphi(u)$ is now

$$
\varphi(u)=-\frac{\frac{\partial p(u)}{\partial u}}{p(u)}=u+\tanh (u) .
$$

The learning rule for supergaussian sources is (equations 2.10 and 2.19):

$$
\Delta \mathbf{W} \propto\left[\mathbf{I}-\tanh (\mathbf{u}) \mathbf{u}^{T}-\mathbf{u} \mathbf{u}^{T}\right] \mathbf{W} .
$$

The difference between the supergaussian learning rule in equation 2.20 and the subgaussian learning rule equation 2.17 is the sign before the tanh function:

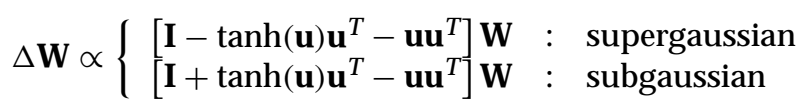

The learning rules differ in the sign before the tanh function and can be determined using a switching criterion. Girolami (1997) employs the sign of kurtosis of the unmixed sources as a switching criterion. However, because there is no general definition for sub- and supergaussian sources, we chose a switching criterion, based on stability criteria, presented in the next subsection.

2.3 Switching Between Nonlinearities. The switching between the suband supergaussian learning rule is

$$
\begin{aligned}
& \Delta \mathbf{W} \propto\left[\mathbf{I}-\mathbf{K} \tanh (\mathbf{u}) \mathbf{u}^{T}-\mathbf{u} \mathbf{u}^{T}\right]
\end{aligned}
$$

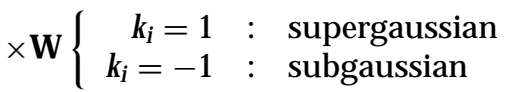




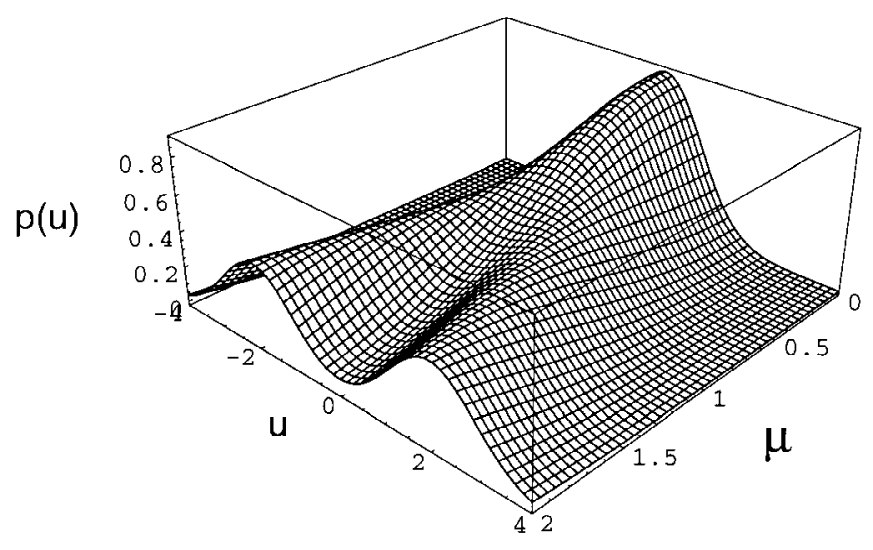

Figure 1: Estimated subgaussian density models for the extended infomax learning rule with $\sigma^{2}=1$ and $\mu_{i}=\{0 \cdots 2\}$. The density becomes clearly bimodal when $\mu_{i}>1$.

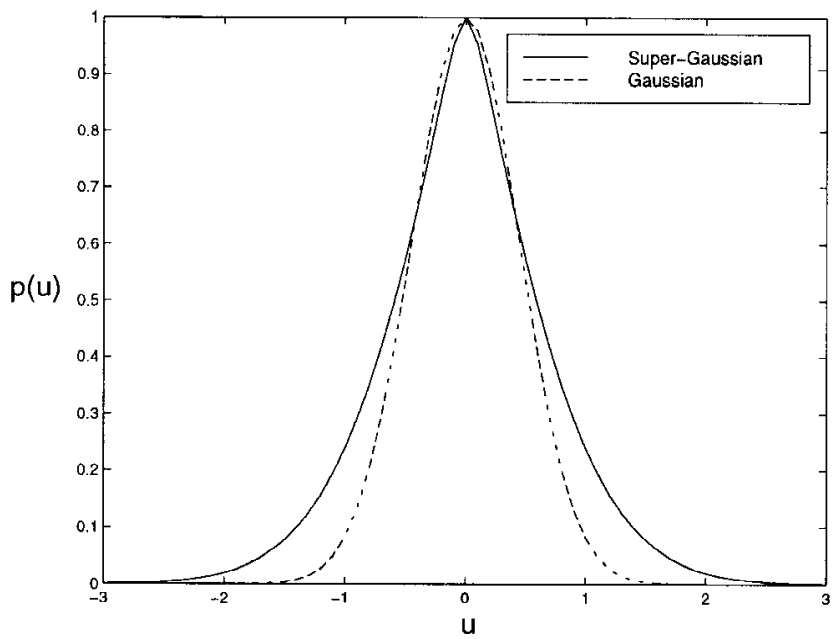

Figure 2: Density model for the supergaussian distribution. The supergaussian model has a heavier tail than the normal density. 
where $k_{i}$ are elements of the $N$-dimensional diagonal matrix $\mathbf{K}$. The switching parameter $k_{i}$ can be derived from the generic stability analysis of separating solutions as employed by Cardoso and Laheld (1996) ${ }^{4}$, Pham and Garrat (1997), and Amari et al. (1997). In the stability analysis, the mean field is approximated by a first-order perturbation in the parameters of the separating matrix. The linear approximation near the stationary point is the gradient of the mean field at the stationary point. The real part of the eigenvalues of the derivative of the mean field must be negative so that the parameters are on average pulled back to the stationary point.

A sufficient condition guaranteeing asymptotic stability can be derived (Cardoso, 1998, in press) so that

$$
\kappa_{i}>0 \quad 1 \leq i \leq N
$$

where $\kappa_{i}$ is

$$
\kappa_{i}=E\left\{\varphi_{i}^{\prime}\left(u_{i}\right)\right\} E\left\{u_{i}^{2}\right\}-E\left\{\varphi_{i}\left(u_{i}\right) u_{i}\right\}
$$

and

$$
\varphi_{i}\left(u_{i}\right)=u_{i}+k_{i} \tanh \left(u_{i}\right) .
$$

Substituting equation 2.25 in equation 2.24 gives

$$
\begin{aligned}
\kappa_{i} & =E\left\{k_{i} \operatorname{sech}^{2}\left(u_{i}\right)+1\right\} E\left\{u_{i}^{2}\right\}-E\left\{\left[k_{i} \tanh \left(u_{i}\right)+u_{i}\right] u_{i}\right\} \\
& =k_{i}\left(E\left\{\operatorname{sech}^{2}\left(u_{i}\right)\right\} E\left\{u_{i}^{2}\right\}-E\left\{\left[\tanh \left(u_{i}\right)\right] u_{i}\right\}\right) .
\end{aligned}
$$

To ensure $\kappa_{i}>0$ the sign of $k_{i}$ must be the same as the sign of $E\left\{\operatorname{sech}^{2}\left(u_{i}\right)\right\} E\left\{u_{i}^{2}\right\}$ $-E\left\{\left[\tanh \left(u_{i}\right)\right] u_{i}\right\}$. Therefore we can use the learning rule in equation 2.22, where the $k_{i}$ 's are

$$
k_{i}=\operatorname{sign}\left(E\left\{\operatorname{sech}^{2}\left(u_{i}\right)\right\} E\left\{u_{i}^{2}\right\}-E\left\{\left[\tanh \left(u_{i}\right)\right] u_{i}\right\}\right)
$$

2.4 The Hyperbolic-Cauchy Density Model. We present another parametric density model that may be used for the separation of sub- and supergaussian sources. We define the parametric mixture density as

$$
p(u) \propto \operatorname{sech}^{2}(u+b)+\operatorname{sech}^{2}(u-b) .
$$

Figure 3 shows the parametric density as a function of $b$. For $b=0$, the parametric density is proportional to the hyperbolic-Cauchy distribution

\footnotetext{
${ }^{4}$ See equations 40 and 41 in their paper.
} 
and is therefore suited for separating supergaussian sources. For $b=2$ the parametric density estimator has a bimodal ${ }^{5}$ distribution with negative kurtosis and is therefore suitable for separating subgaussian sources:

$$
\begin{aligned}
\varphi(u) & =-\frac{\partial}{\partial u} \log p(u) \\
& =-2 \tanh (u)+2 \tanh (u+b)+2 \tanh (u-b) .
\end{aligned}
$$

The learning algorithm for sub- and supergaussian sources is now (equations 2.30 and 2.10)

$$
\Delta \mathbf{W} \propto\left[\mathbf{I}+2 \tanh (\mathbf{u}) \mathbf{u}^{T}-2 \tanh (\mathbf{u}+\mathbf{b}) \mathbf{u}^{T}-2 \tanh (\mathbf{u}-\mathbf{b}) \mathbf{u}^{T}\right] \mathbf{W} .
$$

When $\mathbf{b}=\mathbf{0}$ (where $\mathbf{0}$ is an $N$-dimension vector with elements 0 ), then the learning rule reduces to

$$
\Delta \mathbf{W} \propto\left[\mathbf{I}-2 \tanh (\mathbf{u}) \mathbf{u}^{T}\right] \mathbf{W}
$$

which is exactly the learning rule in Bell and Sejnowski (1995) with the natural gradient extension. For $\mathbf{b}>\mathbf{1}$, the parametric density is bimodal (as shown in Figure 3), and the learning rule is suitable for separating signals with subgaussian distributions. Here again we may use the sign of the general stability criteria in equation 2.23 and $\kappa_{i}$ in equation 2.24 to determine $b_{i}$ so that we can switch between $b_{i}=0$ and, for example, $b_{i}=2$. In Figure 4 we compare the range of kurtosis values of the parametric mixture density models in equations 2.12 and 2.29. The kurtosis value is shown as a function of the shaping parameter $\mu$ for the symmetric Pearson density model and $b$ for the hyperbolic-Cauchy mixture density model. The kurtosis for the Pearson model is strictly negative except for $\mu=0$ when the kurtosis is zero. Because the kurtosis for the hyperbolic-Cauchy model ranges from positive to negative, it may be used to separate signals with both sub- and supergaussian densities.

\section{Simulations and Experimental Results}

Extensive simulations and experiments were performed on recorded data to verify the performance of the extended infomax algorithm equation 2.21. First, we show that the algorithm is able to separate a large number of sources with a wide variety of sub- and supergaussian distributions. We compared the performance of the extended infomax learning rule in equa-

\footnotetext{
${ }^{5}$ Symmetric bimodal densities considered in this article are subgaussian; however, this is not always the case.
} 


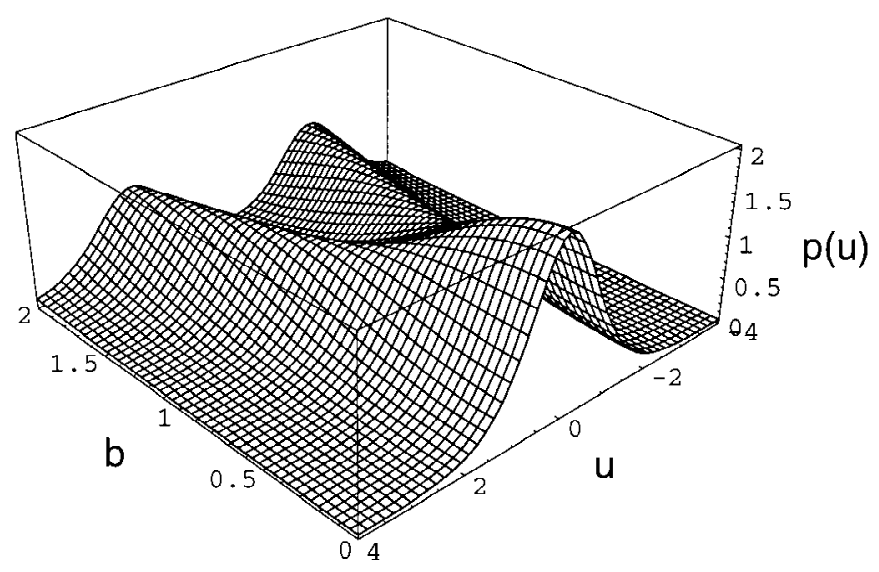

Figure 3: $p(u)$ as a function of $b$. For $b=0$ the density estimate is suited to separate supergaussian sources. If, for example, $b=2$ the density estimate is bimodal and therefore suited to separate subgaussian sources.

tion 2.10 to the original infomax learning rule equation 2.11. Second, we performed a set of experiments on EEG data, which are high dimensional and include various noise sources.

3.1 Ten Mixed Sound Sources. We obtained 10 mixed sound sources that were separated by contextual ICA as described in Pearlmutter and Parra (1996). No prewhitening is required since the transformation $\mathbf{W}$ is not restricted to a rotation, in contrast to nonlinear PCA (Karhunen et al., 1997). All 55,000 data points were passed 20 times through the learning rule using a block size (batch) of 300 . This corresponds to 3666 iterations (weight updates). The learning rate was fixed at 0.0005 . Figure 5 shows the error measure during learning. Both learning rules converged. The small variations of the extended infomax algorithm (upper curve) were due to the adaptation process of $\mathbf{K}$. The matrix $\mathbf{K}$ was initialized to the identity matrix, and during the learning process the elements of $\mathbf{K}$ converge to -1 or 1 to extract sub- or supergaussian sources, respectively. In this simulation example, sources 7, 8, and 9 are close to gaussian, and slight variations of their density estimation change the sign. Annealing of the learning rate reduced the variation. All the music signals had supergaussian distribution and therefore were separable by the original infomax algorithm. The sources are already well separated after one pass through the data (about $10 \mathrm{sec}$ on a SPARC 10 workstation using MATLAB) as shown in Table 1. 


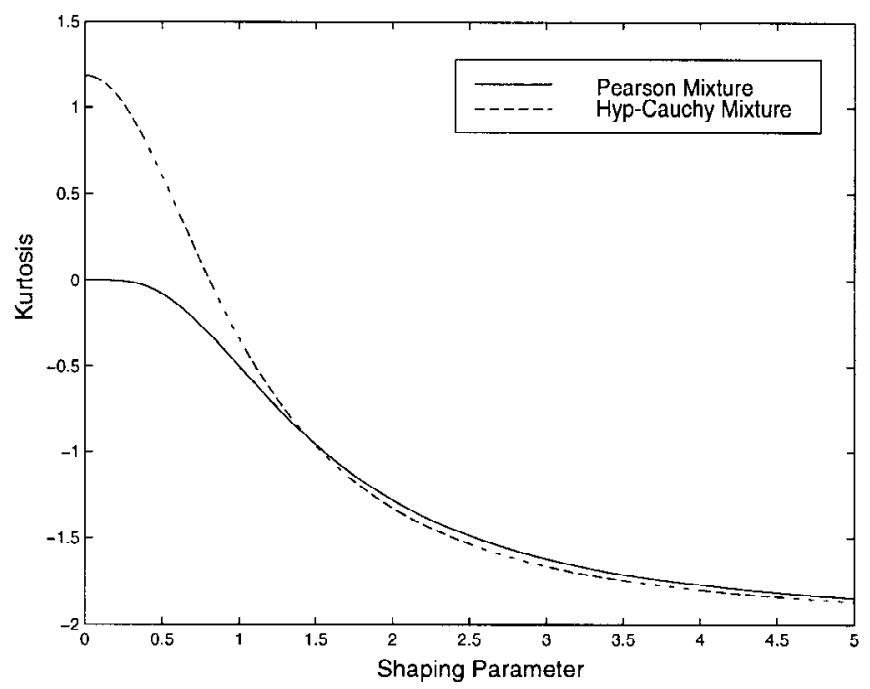

Figure 4: The kurtosis value is shown as a function of the shaping parameter $\mu$ and $b$ ( $\mu$ for the Pearson density model and $b$ for the hyperbolic-Cauchy density model). Both models approach $k_{4}=-2$ as the shaping parameter increases. The kurtosis for the Pearson model is strictly negative except for $\mu=0$. The kurtosis for the hyperbolic-Cauchy model ranges from positive to negative so that we may use this single parametric model to separate signals with sub- and supergaussian densities.

Table 1: Performance Matrix P (Equation 3.2) for 10 Mixed Sound Sources after One Pass through the Data.

\begin{tabular}{rrrrrrrrrr}
\hline-0.09 & -0.38 & 0.14 & -0.10 & -0.06 & 0.93 & -0.36 & -0.54 & 0.17 & 14.79 \\
11.18 & -0.01 & 0.14 & 0.05 & -0.08 & 0.02 & 0.07 & 0.21 & -0.12 & -0.68 \\
0.15 & 0.078 & -0.08 & -0.02 & 10.19 & -0.02 & 0.15 & 0.05 & 0.07 & 0.17 \\
0.39 & 0.61 & -0.70 & -0.07 & 0.14 & 0.32 & -0.08 & 0.85 & 7.64 & -0.16 \\
0.04 & 0.76 & 14.89 & 0.03 & 0.03 & -0.17 & 0.18 & -0.31 & -0.19 & 0.04 \\
0.11 & 12.89 & -0.54 & -0.23 & -0.43 & -0.21 & -0.12 & 0.05 & 0.07 & 0.18 \\
0.45 & 0.16 & -0.02 & 6.53 & 0.24 & 0.98 & -0.39 & -0.97 & 0.06 & -0.08 \\
0.31 & 0.14 & 0.23 & 0.03 & -0.14 & -17.25 & -0.39 & -0.25 & 0.19 & 0.39 \\
-0.54 & -0.81 & 0.62 & 0.84 & -0.18 & 0.47 & -0.04 & 10.48 & -0.92 & 0.12 \\
-0.08 & -0.26 & 0.15 & -0.10 & 0.49 & 0.01 & -10.25 & 0.59 & 0.33 & -0.94 \\
\hline
\end{tabular}

Note: After one pass through the data $\mathbf{P}$ are already close to the identity matrix after rescaling and reordering. An italicized entry signifies the largest component for the corresponding channel. 


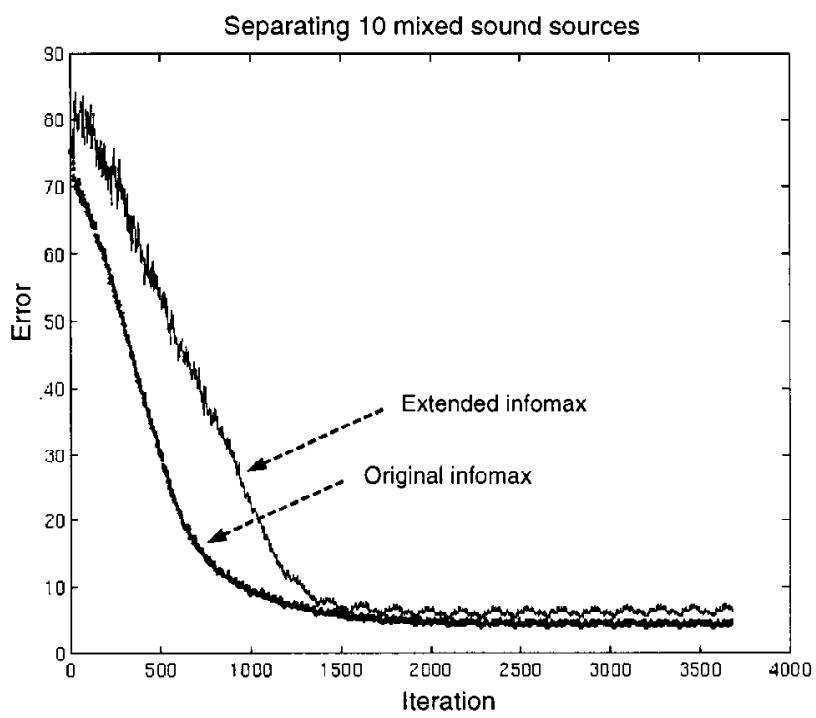

Figure 5: Error measure $E$ in equation 3.2 for the separation of 10 sound sources. The upper curve is the performance for extended infomax, and the lower curve shows the performance for the original infomax. The separation quality is shown in Table 1.

For all experiments and simulations, a momentum term helped to accelerate the convergence of the algorithm:

$$
\Delta \mathbf{W}(n+1)=(1-\alpha) \Delta \mathbf{W}(n)+\alpha \mathbf{W}(n),
$$

where $\alpha$ takes into account the history of $\mathbf{W}$ and $\alpha$ can be increased with an increasing number of weight updates (as $n \rightarrow \infty, \alpha \rightarrow 1$ ).

The performance during the learning process we monitored by the error measure proposed by Amari et al. (1996),

$$
E=\sum_{i=1}^{N}\left(\sum_{j=1}^{N} \frac{\left|p_{i j}\right|}{\max _{k}\left|p_{i k}\right|}-1\right)+\sum_{j=1}^{N}\left(\sum_{i=1}^{N} \frac{\left|p_{i j}\right|}{\max _{k}\left|p_{k j}\right|}-1\right),
$$

where $p_{i j}$ are elements of the performance matrix $\mathbf{P}=$ WA. $\mathbf{P}$ is close to a permutation of the scaled identity matrix when the sources are separated. Figure 5 shows the error measure during the learning process.

To compare the speed of the extended infomax algorithm with another closely related one, we separated the 10 mixed sound sources using the extended exploratory projection pursuit network with inhibitory lateral connections (Girolami \& Fyfe, 1997a). The single feedforward neural network 
converged several times faster than this architecture using the same learning rate and a block size of 1. Larger block sizes can be used in the feedforward network but not the feedback networks, which increase the convergence speed considerably due to a more reliable estimate of the switching matrix $\mathbf{K}$.

3.2 Twenty Mixed Sound Sources. We separated the following 20 sources: 10 soundtracks obtained from Pearlmutter, 6 speech and sound signals used in Bell and Sejnowski (1995), 3 uniformly distributed subgaussian noise signals, and 1 noise source with a gaussian distribution. The densities of the mixtures were close to the gaussian distributions. The following parameters were used: learning rate fixed at 0.0005 , block size of 100 data points, and 150 passes through the data (41,250 iterations). Table 2 compares the separation quality between the infomax algorithm and the extended infomax algorithm.

Figure 6 shows the performance of the matrix $\mathbf{P}$ after the rows were manually reordered and normalized to unity. $P$ is close to the identity matrix, and its off-diagonal elements indicate the amount of error. In this simulation, we employ $k_{4}$ as a measure of the recovery of the sources. The original infomax algorithm separated most of the positive kurtotic sources. However, it failed to extract several sources, including two supergaussian sources (music 7 and 8 ) with low kurtosis ( 0.78 and 0.46 , respectively). In contrast, Figure 7 shows that the performance matrix $\mathbf{P}$ for the extended infomax algorithm is close to the identity matrix. In a listening test, there was a clear separation of all sources from their mixtures. Note that although the sources ranged from Laplacian distributions $(p(s) \propto \exp (-|s|)$, e.g., speech), and gaussian noise to uniformly distributed noise, they were all separated using one nonlinearity.

The simulation results suggest that the supergaussian and subgaussian density estimates in equations 2.12 and 2.18 are sufficient to separate the true sources. The learning algorithms in equations 2.21 and 2.31 performed almost identically.

3.3 EEG Recordings. EEG recordings of brain electrical activity from the human scalp, artifacts such as line noise, eye movements, blinks, and cardiac signals (EKG) pose serious problems in analyzing and interpreting the recordings. Regression methods have been used to remove eye movement partially from the EEG data (Berg \& Scherg, 1991); other artifacts such as electrode noise, cardiac signals, and muscle noise are even more difficult to remove. Recently, Makeig, Bell, Jung, \& Sejnowski (1996) applied ICA to the analysis of EEG data using the original infomax algorithm. They showed that some artifactual components can be isolated from overlapping EEG signals, including alpha and theta bursts.

We analyzed EEG data that were collected to develop a method of objectively monitoring the alertness of operators listening for auditory signals 
Table 2: Kurtosis of the Original Signal Sources and Recovered Signals.

\begin{tabular}{clcccc}
\hline $\begin{array}{c}\text { Source } \\
\text { Number }\end{array}$ & $\begin{array}{l}\text { Source } \\
\text { Type }\end{array}$ & $\begin{array}{c}\text { Original } \\
\text { Kurtosis }\end{array}$ & $\begin{array}{c}\text { Recovered Kurtosis } \\
\text { (infomax) }\end{array}$ & $\begin{array}{c}\text { Recovered Kurtosis } \\
\text { (extended infomax) }\end{array}$ & $\begin{array}{c}\text { Signal-to-Noise Ratio (SNR) } \\
\text { (extended infomax) }\end{array}$ \\
\hline 1 & Music 1 & 2.4733 & 2.4754 & 2.4759 & 43.4 \\
2 & Music 2 & 1.5135 & 1.5129 & 1.5052 & 55.2 \\
3 & Music 3 & 2.4176 & 2.4206 & 2.4044 & 44.1 \\
4 & Music 4 & 1.076 & 1.0720 & 1.0840 & 41.7 \\
5 & Music 5 & 1.0317 & 1.0347 & 1.0488 & 48.1 \\
6 & Music 6 & 1.8626 & 1.8653 & 1.8467 & 32.7 \\
7 & Music 7 & 0.7867 & 0.8029 & 0.7871 & 29.4 \\
8 & Music 8 & 0.4639 & 0.2753 & 0.4591 & 36.4 \\
9 & Music 9 & 0.5714 & 0.5874 & 0.5733 & 46.4 \\
10 & Music 10 & 2.6358 & 2.6327 & 2.6343 & 5.3 \\
11 & Speech 1 & 6.6645 & 6.6652 & 6.6663 & 48.1 \\
12 & Speech 2 & 3.3355 & 3.3389 & 3.3324 & 50.5 \\
13 & Music 11 & 1.1082 & 1.1072 & 1.1053 & 52.6 \\
14 & Speech 3 & 7.2846 & 7.2828 & 7.2875 & 57.1 \\
15 & Music 12 & 2.8308 & 2.8198 & 2.8217 & 61.4 \\
16 & Speech 4 & 10.8838 & 10.8738 & 10.8128 & 67.7 \\
17 & Uniform noise 1 & -1.1959 & -0.2172 & -1.1955 & 63.6 \\
18 & Uniform noise 2 & -1.2031 & -0.2080 & -1.2013 & 24.9 \\
19 & Uniform noise 3 & -1.1966 & -0.2016 & -1.1955 & -0.0399 \\
20 & Gaussian noise & -0.0148 & -0.0964 & & \\
\hline
\end{tabular}

Note: The source signals range from highly kurtotic speech signals, gaussian noise (kurtosis is zero) to noise sources with uniform distribution (negative kurtosis). The sources that failed to separate clearly are italicized. In addition, the SNR is computed for extended infomax. 


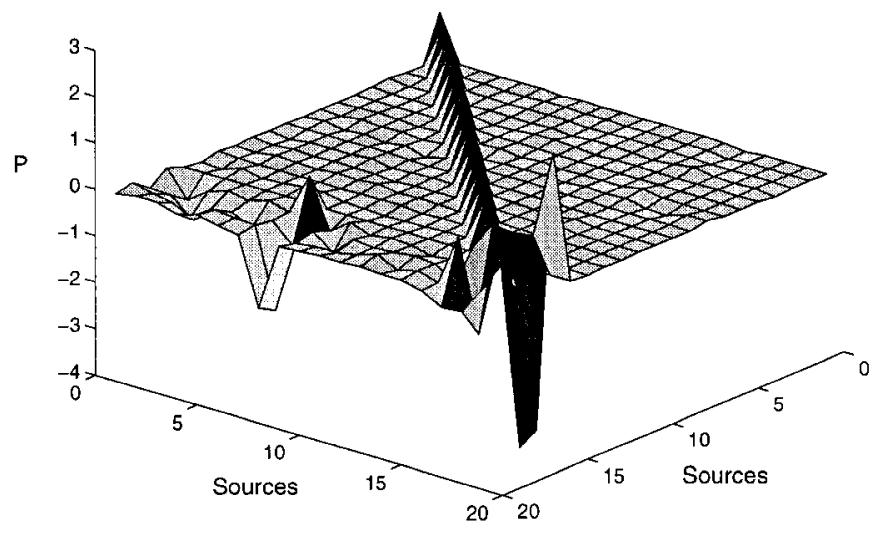

Figure 6: Performance matrix $\mathbf{P}$ for the separation of 20 sources using the original infomax algorithm after normalizing and reordering. Most supergaussian sources were recovered. However, the three subgaussian sources $(17,18,19)$, the gaussian source $(20)$, and two supergaussian sources $(7,8)$ remain mixed and aliased in other sources. In total, 14 sources were extracted, and 6 channels remained mixed. See Table 2.

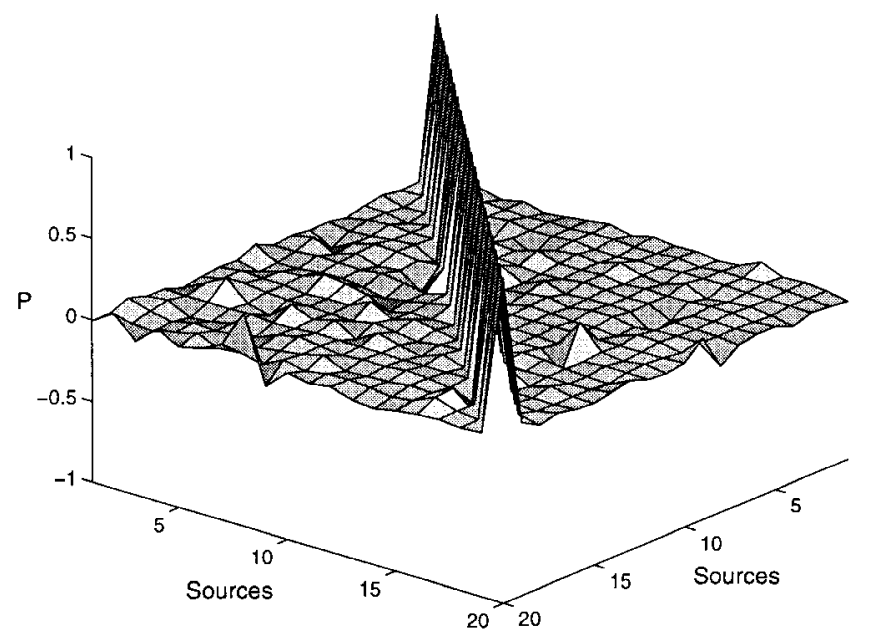

Figure 7: Performance matrix $\mathbf{P}$ for the separation of 20 sources using the extended infomax algorithm after normalizing and reordering. $\mathbf{P}$ is approximately the identity matrix that indicates nearly perfect separation. 
(Makeig \& Inlow, 1993). During a half-hour session, the subject was asked to push a button whenever he or she detected an auditory target stimulus. EEG was collected from 14 electrodes located at sites of the International 1020 System (Makeig et al., 1997) at a sampling rate of $312.5 \mathrm{~Hz}$. The extended infomax algorithm was applied to the 14 channels of 10 seconds of data with the following parameters: learning rate fixed at 0.0005, 100 passes with block size of 100 (3125 weight updates). The power spectrum was computed for each channel, and the power in a band around $60 \mathrm{~Hz}$ was used to compute the relative power for each channel and each separated component.

Figure 8 shows the time course of 14 channels of EEG and Figure 9 the independent components found by the extended infomax algorithm. Several observations on the ICA components in Figure 9 and its power spectrum are of interest:

- Alpha bursts (about $11 \mathrm{~Hz}$ ) were detected in components 1 and 5. Alpha band activity $(8-12 \mathrm{~Hz})$ occurs most often when the eyes are closed and the subject is relaxed. Most subjects have more than one alpha rhythm, with somewhat different frequencies and scalp patterns.

- Theta bursts (about $7 \mathrm{~Hz}$ ) were detected in components 4, 6, and 9 . Theta-band rhythms (4-8 Hz) may occur during drowsiness and transient losses of awareness or microsleeps (Makeig \& Inlow, 1993), but frontal theta bursts may occur during intense concentration.

- An eye blink was isolated in component 2 at 8 sec.

- Line noise of $60 \mathrm{~Hz}$ was concentrated in component 3 (see the bottom of Figure 10).

Figure 10 (top) shows power near $60 \mathrm{~Hz}$ distributed in all EEG channels but predominantly in components 4,13 , and 14 . Figure 10 (middle) shows that the original infomax cannot concentrate the line noise into one component. In contrast, extended infomax (figure 10, bottom panel) concentrates it mainly in one subgaussian component, channel 3.

Figure 11 shows another EEG data set with 23 channels, including 2 EOG (electrooculogram) channels. The eye blinks near $5 \mathrm{sec}$ and $7 \mathrm{sec}$ contaminated all of the channels. Figure 12 shows the ICA components without normalizing the components with respect to their contribution to the raw data. ICA component 1 in Figure 12 contained the pure eye blink signal. Small periodic muscle spiking at the temporal sites (T3 and T4) was extracted into ICA component 14.

Experiments with several different EEG data sets confirmed that the separation of artifactual signals was highly reliable. In particular, severe line noise signals could always be decomposed into one or two components with subgaussian distributions. Jung et al. (1998) show further that eye movement also can be extracted. 


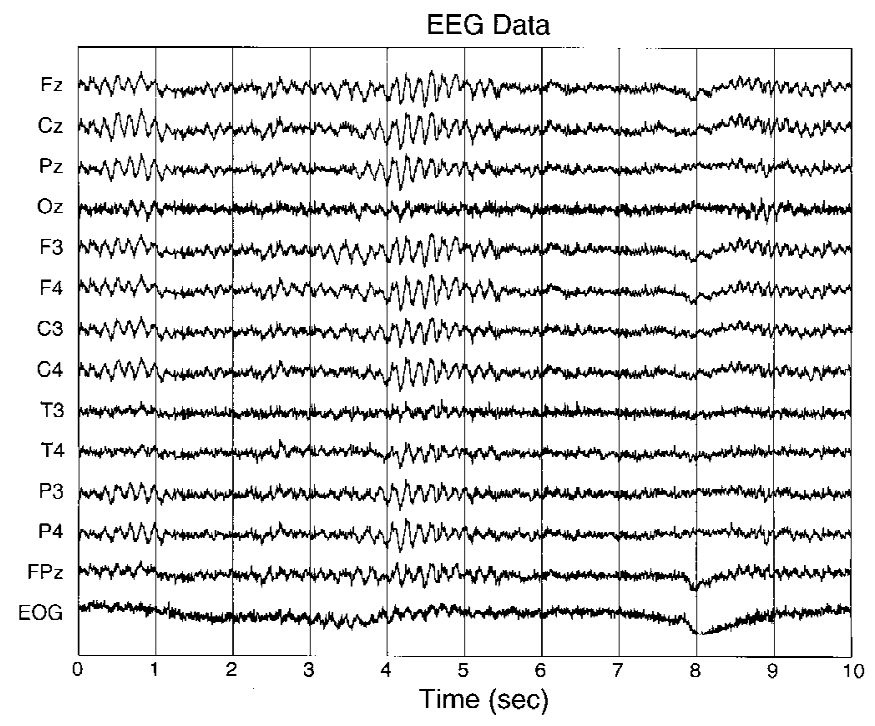

Figure 8: A 10 sec portion of the EEG time series with prominent alpha rhythms (8-21 Hz). The location of the recording electrode from the scalp is indicated on the left of each trace. The electrooculogram (EOG) recording is taken from the temples.

\section{Discussion}

4.1 Applications to Real-World Problems. The results reported here for the separation of eye movement artifacts from EEG recordings have immediate application to medical and research data. Independently, Vigario, Hyvaerinen, and Oja (1996) reported similar findings for EEG recordings using a fixed-point algorithm for ICA (Hyvaerinen \& Oja, 1997). It would be useful to compare this and other ICA algorithms on the same data sets to assess their merits. Compared to traditional techniques in EEG analysis, extended infomax requires less supervision and is easy to apply (see Makeig et al., 1997; Jung et al., 1998). In addition to the very encouraging results on EEG data given here, McKeown et al. (1998) have demonstrated another successful use of the extended infomax algorithm on fMRI recordings. They investigated task-related human brain activity in fMRI data. In this application, they considered both spatial and temporal ICA and found that the extended infomax algorithm extracted subgaussian temporal components that could not be extracted with the original infomax algorithm. 


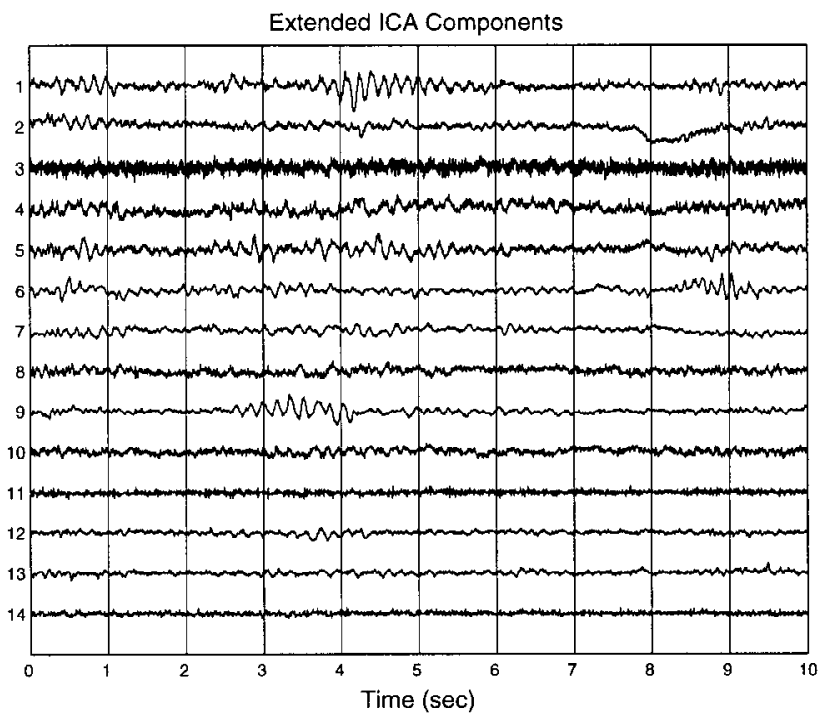

Figure 9: The 14 ICA components extracted from the EEG data in Figure 8. Components $3,4,7,8$, and 10 have subgaussian distributions, and the others have supergaussian distributions. There is an eye movement artifact at 8 seconds. Line noise is concentrated in component 3 . The prominent rhythms in components $1,4,5,6$, and 9 have different time courses and scalp distributions.

4.2 Limitations and Future Research. The extended infomax learning algorithm makes several assumptions that limit its effectiveness. First, the algorithm requires the number of sensors to be the same as or greater than the number of sources $(N \geq M)$. The case when there are more sources than sensors, $N<M$, is of theoretical and practical interest. Given only one or two sensors that observe more than two sources, can we still recover all sources? Preliminary results by Lewicki and Sejnowski (1998) suggest that an overcomplete representation of the data to some extent can extract the independent components using a priori knowledge of the source distribution. This has been applied by Lee, Lewicki, Girolami, and Sejnowski (in press $b$ ) to separate three sources from two sensors.

Second, researchers have recently tackled the problem of nonlinear mixing phenomena. Yang, Amari, and Cichocki (1997), Taleb and Jutten (1997), and Lee, Koehler, and Orglmeister (1997) propose extensions when linear mixing is combined with certain nonlinear mixing models. Other approaches use self-organizing feature maps to identify nonlinear features in the data (Lin \& Cowan, 1997; Pajunen \& Karhunen, 1997). Hochreiter and 

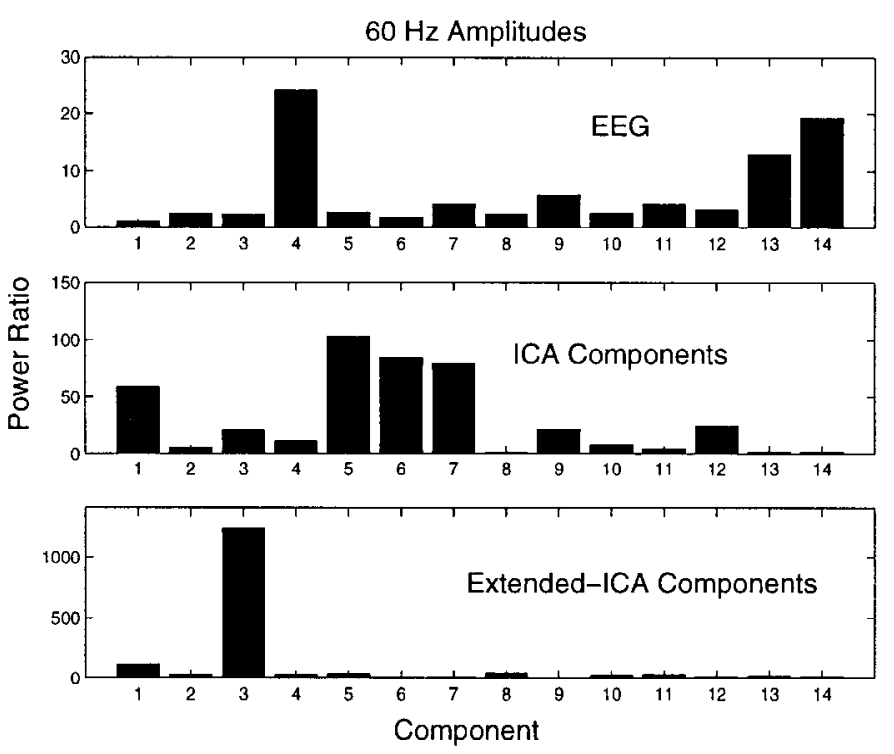

Figure 10: (Top) Ratio of power near $60 \mathrm{~Hz}$ over 14 components for EEG data in Figure 8. (Middle) Ratio of power near $60 \mathrm{~Hz}$ for the 14 infomax ICA components. (Bottom) Ratio of power near $60 \mathrm{~Hz}$ for the 14 extended infomax ICA components in Figure 9. Note the difference in scale by a factor of 10 between the original infomax and the extended infomax.

Schmidhuber (1999) have proposed low-complexity coding and decoding approaches for nonlinear ICA.

Third, sources may not be stationary; sources may appear and disappear and move (as when a speaker moves in a room). In these cases, the weight matrix $\mathbf{W}$ may change completely from one time point to the next. This is a challenging problem for all existing ICA algorithms. A method to model the context switching (nonstationary mixing matrix) in an unsupervised way is proposed in Lee, Lewicki, and Sejnowski (1999).

Fourth, sensor noise may influence separation and should be included in the model (Nadal \& Parga 1994; Moulines et al., 1997; Attias \& Schreiner, 1999). Much more work needs to be done to determine the effect of noise on performance.

In addition to these limitations, there are other issues that deserve further research. In particular, it remains an open question to what extent the learning rule is robust to parametric mismatch given a limited number of data points.

Despite these limitations, the extended infomax ICA algorithm presented here should have many applications where both subgaussian and super- 


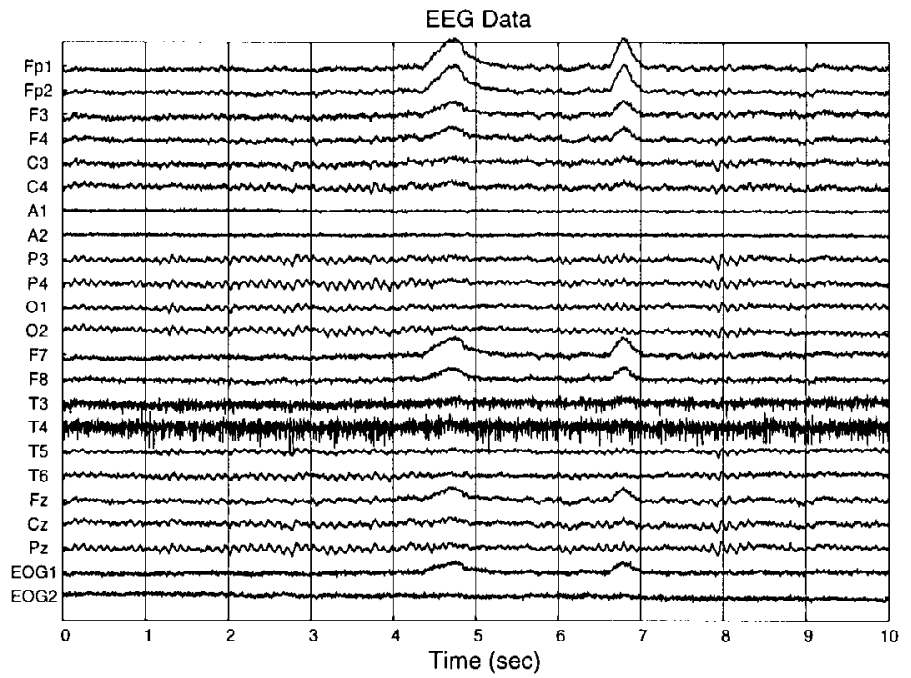

Figure 11: EEG data set with 23 channels including 2 EOG channels. Note that at around 4-5 sec and 6-7 sec, artifacts from severe eye blinks contaminate the data set.

gaussian sources need to be separated without additional prior knowledge of their statistical properties.

\section{Conclusions}

The extended infomax ICA algorithm proposed here is a promising generalization that satisfies a general stability criterion for mixed subgaussian and supergaussian sources (Cardoso \& Laheld, 1996). Based on the learning algorithm first derived by Girolami (1997) and the natural gradient, the extended infomax algorithm has shown excellent performance on several large real data sets derived from electrical and blood flow measurements of functional activity in the brain. Compared to the originally proposed infomax algorithm (Bell and Sejnowski, 1995), the extended infomax algorithm separates a wider range of source signals while maintaining its simplicity.

Acknowledgments

T. W. L. was supported by the German Academic Exchange Program. M. G. was supported by a grant from NCR Financial Systems (Ltd.), Knowledge Laboratory, Advanced Technology Development Division, Dundee, Scotland. T. J. S. was supported by the Howard Hughes Medical Institute. We 


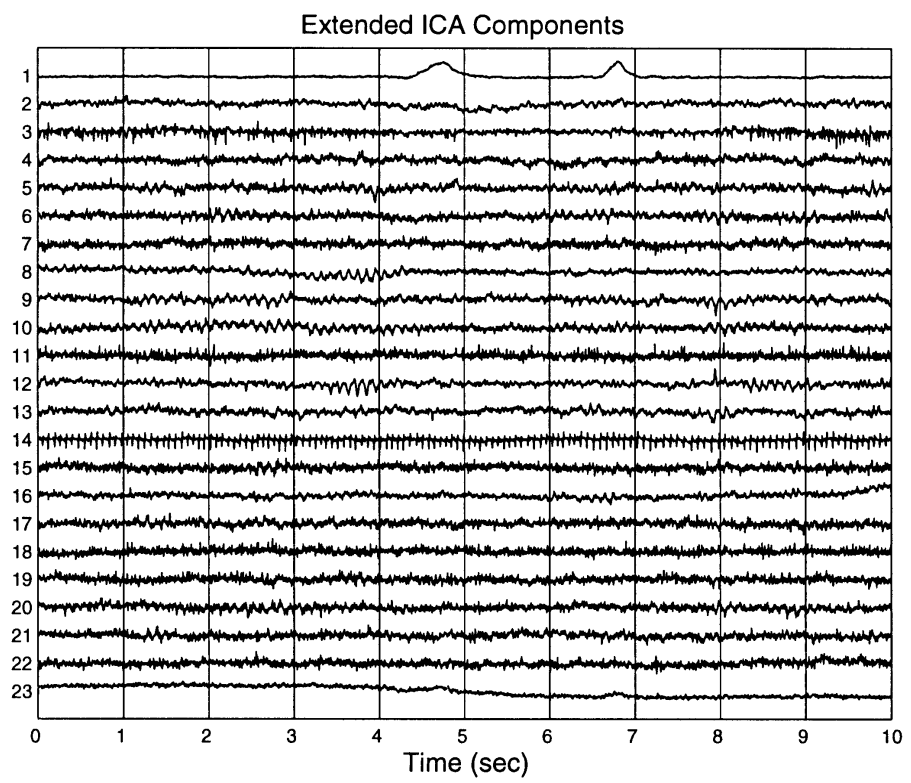

Figure 12: Extended infomax ICA components derived from the EEG recordings in Figure 11. The eye blinks are clearly concentrated in component 1 . Component 14 contains the steady-state signal.

are much indebted to Jean-François Cardoso for insights and helpful comments on the stability criteria and Tony Bell for general comments and discussions. We are grateful to Tzyy-Ping Jung and Scott Makeig for EEG data, as well as useful discussions and comments, and to Olivier Coenen for helpful comments. We thank the reviewers for fruitful comments.

\section{References}

Amari, S. (1998). Natural gradient works efficiently in learning. Neural Computation, 10, 251-276.

Amari, S., \& Cardoso, J.-F. (1997). Blind source separation-Semiparametric statistical approach. IEEE Trans. on Signal Processing, 45(11), 2692-2700.

Amari, S., Chen, T.-P., \& Cichocki, A. (1997). Stability analysis of adaptive blind source separation. Neural Networks, 10(8), 1345-1352.

Amari, S., Cichocki, A., \& Yang, H. (1996). A new learning algorithm for blind signal separation. In D. Touretzky, M. Mozer, \& M. Hasselmo (Eds.), Advances in neural information processing systems, 8 (pp. 757-763). Cambridge, MA: MIT Press.

Attias, H. (1999). Blind separation of noisy mixtures: An EM algorithm for factor analysis. Neural Computation, 11, to appear in 11-4. 
Bell, A. J., \& Sejnowski, T. J. (1995). An information-maximization approach to blind separation and blind deconvolution. Neural Computation, 7, 11291159.

Berg, P., \& Scherg, M. (1991). Dipole models of eye movements and blinks. Electroencephalog. Clin. Neurophysiolog., 79, 36-44.

Cardoso, J.-F. (1998). Blind signal separation: Statistical principles. Proceedings of IEEE, 86(10), 2009-2025.

Cardoso, J.-F. (in press). Unsupervised adaptive filtering. InS. Haykin (Ed.), Entropic contrasts for source separation. Englewood Cliffs, NJ: Prentice Hall.

Cardoso, J.-F. (1997). Infomax and maximum likelihood for blind source separation. IEEE Signal Processing Letters, 4(4), 112-114.

Cardoso, J.-F., \& Laheld, B. (1996). Equivariant adaptive source separation. IEEE Trans. on S.P., 45(2), 434-444.

Cardoso, J., \& Soloumiac, A. (1993). Blind beamforming for non-gaussian signals. IEE Proceedings- $F, 140(46), 362-370$.

Cichocki, A., Unbehauen, R., \& Rummert, E. (1994). Robust learning algorithm for blind separation of signals. Electronics Letters, 30(17), 1386-1387.

Comon, P. (1994). Independent component analysis-A new concept? Signal Processing, 36(3), 287-314.

Cover, T., \& Thomas, J. (Eds.). (1991). Elements of information theory. New York: Wiley.

Deco, G., \& Obradovic, D. (1996). An information-theoretic approach to neural computing. Berlin: Springer-Verlag.

Gaeta, M., \& Lacoume, J.-L. (1990). Source separation without prior knowledge: The maximum likelihood solution. Proc. EUSIPO (pp. 621-624).

Girolami, M. (1997). Self-organizing artificial neural networks for signal separation. Unpublished Ph.D. dissertation, Paisley University, Scotland.

Girolami, M. (1998). An alternative perspective on adaptive independent component analysis algorithms. Neural Computation, 10, 2103-2114.

Girolami, M., \& Fyfe, C. (1997a). Extraction of independent signal sources using a deflationary exploratory projection pursuit network with lateral inhibition. I.E.E Proceedings on Vision, Image and Signal Processing Journal, 14(5), 299-306.

Girolami, M., \& Fyfe, C. (1997b). Generalised independent component analysis through unsupervised learning with emergent bussgang properties. In Proc. ICNN (pp. 1788-1891). Houston, TX.

Hochreiter, S., \& Schmidhuber, J. (1999). Feature extraction through LOCOCODE. Neural Computation, 11, to appear in 11-3.

Hyvaerinen, A., \& Oja, E. (1997). A fast fixed-point algorithm for independent component analysis. Neural Computation, 9, 1483-1492.

Jung, T.-P., Humphries, C., Lee, T.-W., Makeig, S., McKeown, M., Iragui, V., \& Sejnowski, T. J. (1998). Extended ICA removes artifacts from electroencephalographic recordings. In M. Kearns, M. Jordan, \& S. Solla (Eds.), Advances in neural information processing systems, 10 (pp. 894-900). Cambridge, MA: MIT Press.

Jutten, C., \& Hérault, J. (1991). Blind separation of sources, part I: An adaptive algorithm based on neuromimetic architecture. Signal Processing , 24, 1-10.

Karhunen, J. (1996). Neural approaches to independent component analysis 
and source separation. In Proc. 4th European Symposium on Artificial Neural Networks (pp. 249-266). Bruges, Belgium.

Karhunen, J., Oja, E., Wang, L., Vigario, R., \& Joutsensalo, J. (1997). A class of neural networks for independent component analysis. IEEE Trans. on Neural Networks, 8, 487-504.

Lee, T.-W. (1998). Independent component analysis: Theory and applications. Dordrecht: Kluwer Academic Publishers, ISBN: 0-7923-8261-7.

Lee, T.-W., Girolami, M., Bell, A. J., \& Sejnowski, T. J. (1999). A unifying framework for independent component analysis. Computers and Mathematics with Applications, in press.

Lee, T.-W., Koehler, B., \& Orglmeister, R. (1997). Blind separation of nonlinear mixing models. In IEEE NNSP (pp. 406-415). Florida.

Lee, T.-W., Lewicki, M. S., Girolami, M., \& Sejnowski, T. J. (in press). Blind source separation of more sources than mixtures using overcomplete representations. IEEE Signal Processing Letters.

Lee, T.-W., Lewicki, M. S., and Sejnowski, T. J. (1999). Unsupervised classification with non-gaussian mixture models using ICA. In Advances in neural information processing systems, 11, Cambridge, MA: MIT Press.

Lewicki, M., \& Sejnowski, T. J. (1998). Learning nonlinear overcomplete representations for efficient coding. In M. Kearns, M. Jordan, \& S. Solla (Eds.), Advances in neural information processing systems, 10 (pp. 815-821). Cambridge, MA: MIT Press.

Lin, J., \& Cowan, J. (1997). Faithful representation of separable input distributions. Neural Computation, 9, 6:1305-1320.

Makeig, S., Bell, A. J., Jung, T., \& Sejnowski, T. J. (1996). Independent component analysis of electroencephalographic data. In D. Touretzky, M. Moser, \& M. Hasselmo (Eds.), Advances in neural information processing systems, 8 (pp. 145-151). Cambridge, MA: MIT Press.

Makeig, S., \& Inlow, M. (1993). Changes in the EEG spectrum predict fluctuations in error rate in an auditory vigilance task. Society for Psychophysiology, 28, S39.

Makeig, S., Jung, T., Bell, A. J., Ghahremani, D., \& Sejnowski, T. J. (1997). Blind separation of event-related brain response into spatial independent components. Proceedings of the National Academy of Sciences, 94, 10979-10984.

McKeown, M., Makeig, S., Brown, G., Jung, T.-P., Kindermann, S., Lee, T.-W., \& Sejnowski, T. J. (1998). Spatially independent activity patterns in functional magnetic resonance imaging data during the Stroop color-naming task. Proceedings of the National Academy of Sciences, 95, 803-810.

Moulines, E., Cardoso, J.-F., \& Cassiat, E. (1997). Maximum likelihood for blind separation and deconvolution of noisy signals using mixture models. In Proc. ICASSP'97 (Vol. 5, pp. 3617-3620). Munich.

Nadal, J.-P., \& Parga, N. (1994). Non linear neurons in the low noise limit: A factorial code maximizes information transfer. Network, 5, 565-581.

Nadal, J.-P., \& Parga, N. (1997). Redundancy reduction and independent component analysis: Conditions on cumulants and adaptive approaches. Neural Computation, 9, 1421-1456.

Oja, E. (1997). The nonlinear PCA learning rule in independent component analysis. Neurocomputing, 17, 25-45. 
Pajunen, P., \& Karhunen, J. (1997). A maximum likelihood approach to nonlinear blind source separation. In ICANN (pp. 541-546). Lausanne.

Pearlmutter, B., \& Parra, L. (1996). A context-sensitive generalization of ICA. In International Conference on Neural Information Processing (pp. 151-157).

Pearson, K. (1894). Contributions to the mathematical study of evolution. Phil. Trans. Roy. Soc. A, 185(71).

Pham, D.-T., \& Garrat, P. (1997). Blind separation of mixture of independent sources through a quasi-maximum likelihood approach. IEEE Trans. on Signal Proc., 45(7), 1712-1725.

Roth, Z., \& Baram, Y. (1996). Multidimensional density shaping by sigmoids. IEEE Trans. on Neural Networks, 7(5), 1291-1298.

Stuart, A., \& Ord, J. (1987). Kendall's advanced theory of statistic, 1, Distribution theory. New York: Wiley.

Taleb, A., \& Jutten, C. (1997). Nonlinear source separation: The post-nonlinear mixtures. In ESANN (pp. 279-284).

Vigario, R., Hyvaerinen, A., \& Oja, E. (1996). ICA fixed-point algorithm in extraction of artifacts from EEG. In IEEE Nordic Signal Processing Symposium (pp. 383-386). Espoo, Finland.

Xu, L., Cheung, C., Yang, H., \& Amari, S. (1997). Maximum equalization by entropy maximization and mixture of cumulative distribution functions. In Proc. of ICNN'97 (pp. 1821-1826). Houston.

Yang, H., Amari, S., \& Cichocki, A. (1997). Information back-propagation for blind separation of sources from non-linear mixtures. In Proc. of ICNN (pp. 2141-2146). Houston.

Received August 1, 1997; accepted May 11, 1998. 